\title{
Dynamical Calculation of Multi-Modal Nuclear Fission of Fermium Nuclei
}

\author{
T. Asano, ${ }^{*, a}$ T. Wada, ${ }^{a}$ M. Ohta, ${ }^{\text {a T. Ichikawa, }}{ }^{\text {b }}$ S. Yamaji, ${ }^{c}$ and H. Nakahara ${ }^{d}$ \\ ${ }^{a}$ Department of Physics, Konan University, 8-9-1 Okamoto, Kobe 658-8501, Japan \\ bapan Atomic Energy Research Institute, Tokai-Mura, Ibaraki 319-1195, Japan \\ ${ }^{\mathrm{c} C y c l o t r o n}$ Center, RIKEN, Wako, Saitama 351-0198, Japan \\ ${ }^{\mathrm{d}}$ Graduate School of Science, Tokyo Metropolitan University, Tokyo 192-0397, Japan
}

\section{Received: November 6, 2003; In Final Form: February 20, 2004}

\begin{abstract}
Multi-modal nuclear fission of fermium isotopes at low excitation energy is dynamically investigated. The multidimensional Langevin equation is used for the dynamical calculation. We use the potential energy taking account of the microscopic energy which depends on the excitation energy. We calculate the mass and the total kinetic energy (TKE) distributions of the fission fragments for ${ }^{264} \mathrm{Fm}$. The deformation of the fission fragments at the scission plays an important role in classifying the nuclear fission modes. We show that there exist following three types for the scission configuration classified by the shape of the fission fragments: mass-symmetric compact configuration, mass-asymmetric configuration, and mass-symmetric elongated configuration. We calculate the average mass number and the average TKE for each configuration. We also study the isotope dependence of the mass distribution of the fission fragments from three fermium isotopes.
\end{abstract}

\section{Introduction}

In studying the multi-modal nuclear fission, fermium nuclei are very important from the viewpoint of magicity of the daughter nuclei since they decay into two proton-magic $(Z=50)$ nuclei $\mathrm{Sn}$ in the mass-symmetric fission. In particular, the nucleus ${ }^{264} \mathrm{Fm}$ decays into two identical double-magic $(Z=50$, $N=82$ ) nuclei ${ }^{132} \mathrm{Sn}$.

It was found that there is a strong isotope dependence in the fragment mass distribution of the spontaneous fission of fermium nuclei; a mass-symmetric peak is observed for ${ }^{258,259} \mathrm{Fm}$, while mass-asymmetric peaks are obtained for ${ }^{254,256,257} \mathrm{Fm} .{ }^{1-6}$ For the thermal neutron induced fission of ${ }^{255} \mathrm{Fm}$, which should be compared with the spontaneous fission of ${ }^{256} \mathrm{Fm}$, a masssymmetric peak is observed in the total mass distribution; the fragment mass distribution depends also on the excitation energy. For the thermal neutron induced fission of ${ }^{255} \mathrm{Fm}$, the authors of Reference 1 cut the total kinetic energy (TKE) distribution into several energy bins and studied the mass distribution for each bin. They obtained a sharp mass-symmetric distribution for high TKE bins (TKE > $220 \mathrm{MeV}$ ) while they obtained mass-asymmetric distribution for low TKE bins (TKE $<220 \mathrm{MeV}$ ). Namely, the total mass distribution is composed of at least two components: a mass-symmetric component with higher TKE and a mass-asymmetric component with lower TKE. For the spontaneous fission of ${ }^{258} \mathrm{Fm}$, the authors of Reference 5 fitted the TKE distribution by two Gaussians and found that the high TKE component (TKE $>220 \mathrm{MeV}$ ) corresponds to a sharp mass-symmetric distribution while the low TKE one (TKE < $220 \mathrm{MeV}$ ) corresponds to a broad mass-symmetric distribution. In the both cases of the thermal neutron induced fission of ${ }^{255} \mathrm{Fm}$ and the spontaneous fission of ${ }^{258} \mathrm{Fm}$, the fission events with the high TKE were found to form a narrow masssymmetric distribution.

The mass distribution and the TKE distribution consist of several components and these components correspond to different nuclear fission modes. ${ }^{1-11}$ Such a phenomenon is observed not only for fermium nuclei but also for other

*Corresponding author. E-mail: dn121001@center.konan-u.ac.jp. FAX: +81-78-439-2539. nuclei. $^{7-11}$ It is known that only the mass-symmetric fission path exists in the potential energy surface (PES) given by the liquid drop model (LDM), so the shell effect plays a crucial role for the multi-modal nuclear fission.

In order to understand the multi-modal nuclear fission, many theoretical efforts have been done so far. One is the method conjecturing the mass distribution from the PES and another is dynamical calculation. The former has given an explanation for the existence of several fission paths for ${ }^{264} \mathrm{Fm} .{ }^{12,13}$ This approach has been applied to many nuclei, but dynamical effects, such as the dissipation, are not included. Dynamical calculations using the multi-dimensional Langevin equation have been applied at high excitation energies and they explain the experimental data such as pre-scission neutron multiplicity. ${ }^{14}$ Since the shell correction energy exists at low excitation energies, e.g. at energies lower than $30 \mathrm{MeV}$, the inclusion of the shell correction energy is very important in the dynamical calculation of the multi-modal nuclear fission. This has been pointed out for the fission of trans-actinide nuclei ${ }^{15}$ and for the fusion-fission mechanism of the superheavy nuclei. ${ }^{16}$ In this paper the dynamical calculation with the potential energy including the shell effects is performed to discuss the multi-modal nuclear fission of fermium isotopes.

Section 2 is a reminder of the multi-dimensional Langevin equation and the potential energy. Calculated results are presented in sec. 3. Summary is given in sec. 4.

\section{Methods}

Dynamical approach based on the multi-dimensional Langevin equation has been successfully applied to nuclear reactions, i.e., fission and fusion-fission. ${ }^{14-18}$ In this study, we apply this approach to investigate the multi-modal nuclear fission in the fermium nuclei. The time evolution of the fission process in the multi-dimensional deformation space is traced starting from the ground state of the fissioning nucleus via saddle points to various scission configurations.

The multi-dimensional Langevin equation has the following form; 


$$
\begin{aligned}
& \frac{d q_{i}}{d t}=\left(m^{-1}\right)_{i j} p_{j}, \\
& \frac{d p_{i}}{d t}=-\frac{\partial V_{i}}{\partial q_{i}}-\frac{1}{2} \frac{\partial}{\partial q_{i}}\left(m^{-1}\right)_{j k} p_{j} p_{k}-\gamma_{i j}\left(m^{-1}\right)_{j k} p_{k}+g_{i j} R_{j}(t),
\end{aligned}
$$

where $q_{i}$ denotes a coordinate in the deformation space and $p_{i}$ is its conjugate momentum. The summation from 1 to $n$ over the repeated indices is assumed with $n$ being the number of collective degrees of freedom. $V(q)$ is the potential energy and $m_{i j}(q)$ and $\gamma_{i j}(q)$ are the shape-dependent collective inertia and dissipation tensors, respectively. The hydrodynamical inertia tensor is adopted with the Werner-Wheeler approximation for the velocity field. ${ }^{19}$ The dissipation tensor is calculated with the one-body type wall-and-window formula. ${ }^{20}$ The normalized random force $R_{i}(t)$ is assumed to be a white noise, i.e.,

$$
\left\langle R_{i}(t)\right\rangle=0,\left\langle R_{i}\left(t_{1}\right) R_{j}\left(t_{2}\right)\right\rangle=2 \delta_{i j} \delta\left(t_{1}-t_{2}\right)
$$

The strength of the random force $g_{i j}$ is given by $\Sigma_{k} g_{i k} g_{j k}=T \gamma_{i j}$, where $T$ is the temperature of the compound nucleus. It is calculated from the excitation energy $E_{\mathrm{X}}$ as $E_{\mathrm{X}}=a T^{2}$, where $a$ is the level density parameter for a spherical nucleus. The potential energy is calculated using the macroscopic-microscopic method. The macroscopic part of the energy is calculated with the Yukawa plus exponential model ${ }^{21}$ and the microscopic part is calculated with the Strutinsky shell correction method ${ }^{22,23}$ using the two-center harmonic oscillator single-particle potential. ${ }^{24-27}$ The potential energy is expressed as a sum of the two terms,

$$
V\left(q, E_{\mathrm{X}}\right)=E_{\mathrm{Macro}}(q)+E_{\mathrm{Micro}}\left(q, E_{\mathrm{X}}\right) .
$$

The macroscopic energy consists of the nuclear potential energy $E_{\text {Nuclear }}$ and the Coulomb energy $E_{\text {Coulomb }}{ }^{21}$ The microscopic energy is calculated as a sum of the shell correction energy $\Delta E_{\mathrm{s}}$ and the pairing correlation correction energy $\Delta E_{\mathrm{pc}}{ }^{28}$ The microscopic energy depends on the excitation energy (temperature) since the occupation probability of the single particle levels depends on the excitation energy. The excitation energy dependence of the microscopic energy is introduced in the following form based on Ignatyuk's suggestion; ${ }^{29}$

$$
E_{\text {Micro }}\left(q, E_{\mathrm{X}}\right)=E_{\text {Micro }}\left(q, E_{\mathrm{X}}=0\right) \exp \left(-E_{\mathrm{X}} / E_{\mathrm{d}}\right),
$$

where $E_{\mathrm{X}}$ is the excitation energy and $E_{\mathrm{d}}$ is the shell damping energy. We take $E_{\mathrm{d}}$ as $20.0 \mathrm{MeV}$ in this study.

The nuclear shape is expressed by three parameters. We

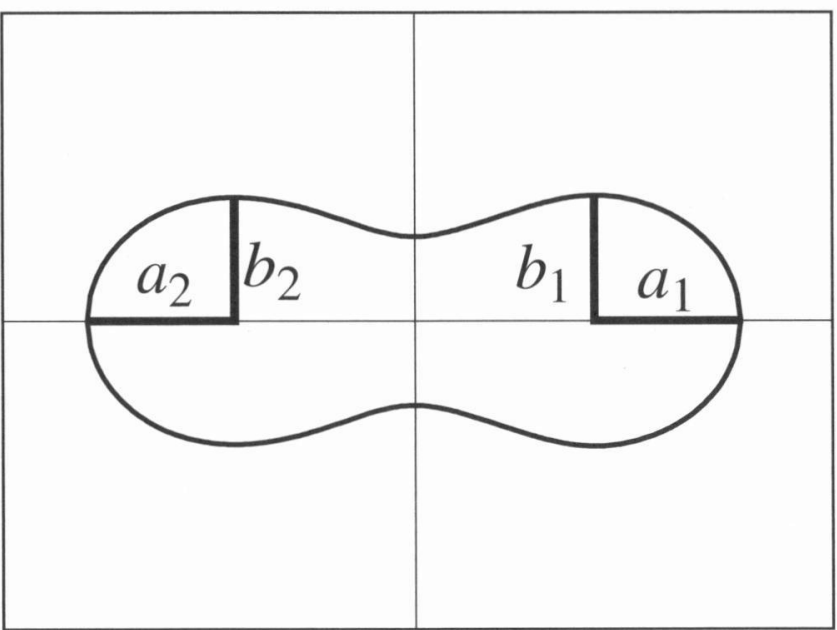

Figure 1. Illustration of $a_{\mathrm{i}}$ and $b_{\mathrm{i}}$ that determine the fragment deformation. treat $R_{\mathrm{C} . \mathrm{M}}$. (the distance between the centers of mass of future fission fragments), $\delta$ (the deformation of the fission fragments), and $A_{1}$ (the mass number of a fission fragment with $A_{2}$ being the mass number of the other fission fragment) as the three collective parameters. In order to obtain the deformation of the two fission fragments by a single parameter $\delta$, we introduce the following relation for the ratio of the two axes $a_{i}$ and $b_{i}$ (see Figure 1):

$$
\frac{a_{i}}{b_{i}}=\frac{2 A_{i}}{A} \frac{3+\delta}{3-2 \delta}(i=1,2),
$$

where $A$ is the mass number of the fissioning nucleus. Figures 2 and 3 show various nuclear shapes that can be described with the three collective parameters. Figure 2 shows the nuclear shapes in the mass-symmetric fission and Figure 3 shows the nuclear shapes for the mass-asymmetric division with $\delta=0.0$.

In the present study, we used TWOCTR of two-center shell model code to calculate the potential energy surface. ${ }^{26,27,30}$ The origin of the potential energy is set so that the macroscopic energy for the spherical shape vanishes. We do not take account of the effect of the angular momentum or the particle evaporation in this study.

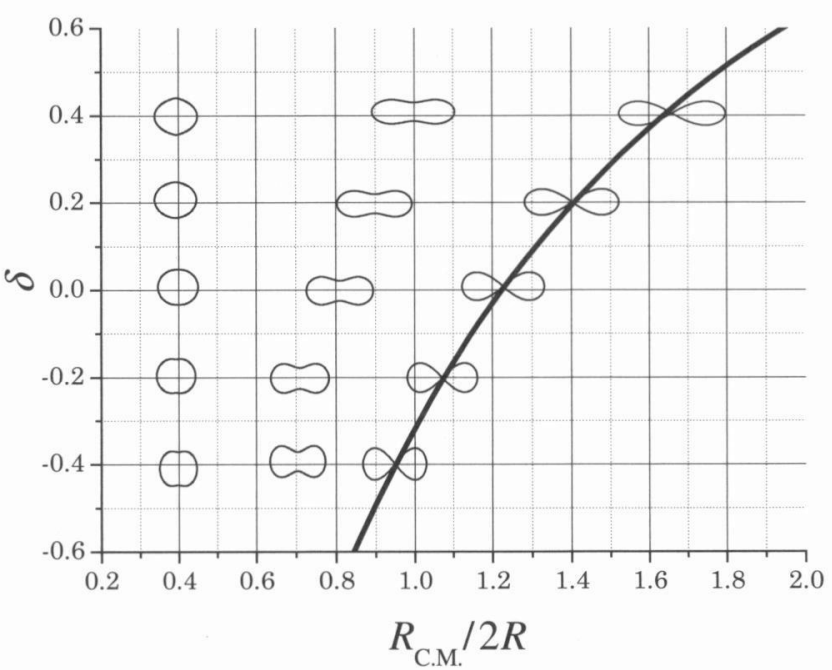

Figure 2. Various nuclear shapes in the deformation space for the mass-symmetric fission of ${ }^{264} \mathrm{Fm}$. The abscissa denotes the distance between the centers of mass of future fission fragments and the ordinate the deformation parameter $\delta$. $R$ is the radius of the parent nucleus. The solid line shows the scission line.

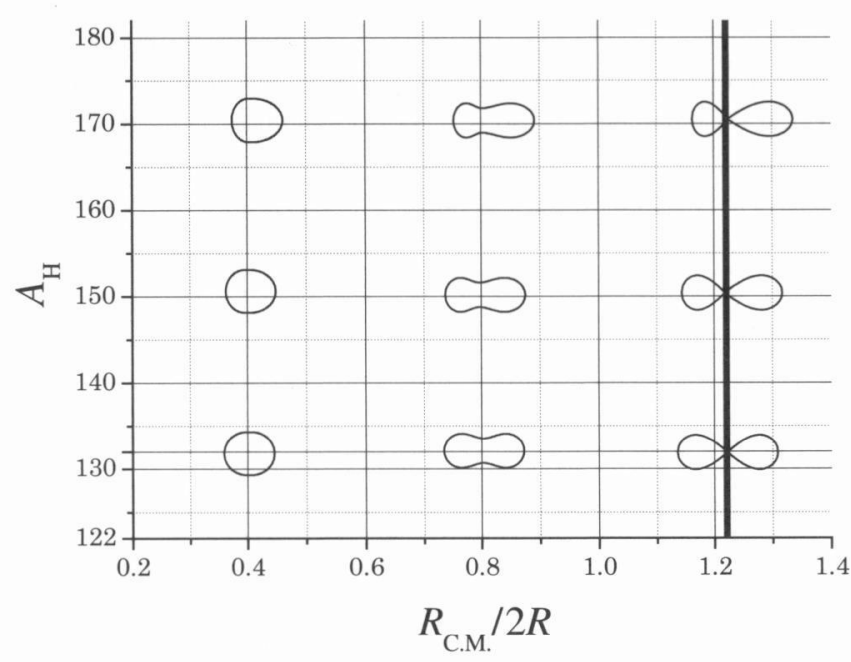

Figure 3. Nuclear shapes in the deformation space with $\delta=0.0$ for ${ }^{264} \mathrm{Fm}$. The abscissa denotes the distance between the centers of mass of future fission fragments and the ordinate the mass number of the heavy fission fragment. $R$ is the radius of the parent nucleus. The solid line shows the scission line. 


\section{Results}

First, we investigate the fission of ${ }^{264} \mathrm{Fm}$. Figures 4 and 5 show the mass distribution and the TKE distribution of the fission fragments at the excitation energy $E_{\mathrm{X}}=10.0 \mathrm{MeV}$. One observes a single peak for the mass distribution and three peaks for the TKE distribution. Since the fission fragments in the mass-symmetric fission of ${ }^{264} \mathrm{Fm}$ are double-magic nuclei, it is expected that the scission configuration is compact. The TKE of the fission fragments essentially depends on the distance between the centers of mass of fission fragments. Thus the TKE of the fission fragments depends sensitively on the compactness of the scission configuration. The masssymmetric compact scission configuration corresponds to the highest TKE. It is expected that the other two TKE peaks correspond to more elongated scission configurations.

As is shown in Figure 2, the compactness of the scission configuration is governed by the fragment deformation parameter $\delta$. Therefore, we pay special attention to the deformation parameter $\delta$ at the scission configuration. In Figure 6 , we show the distribution of the deformation parameter $\delta$ at the scission configuration. From the figure, we observe that this distribution consists of three components similarly to the TKE distribution shown in Figure 5. It is expected that there is a correlation between the structures appearing in Figures 5 and 6. We classify the fission events into three components according to the values of the deformation parameter $\delta$ at the scission configuration; Component I corresponds to $\delta<0.04$, Component II corresponds to $0.04<\delta<0.26$ and Component III corresponds to $\delta>0.26$. In Figure 7 , the TKE distribution

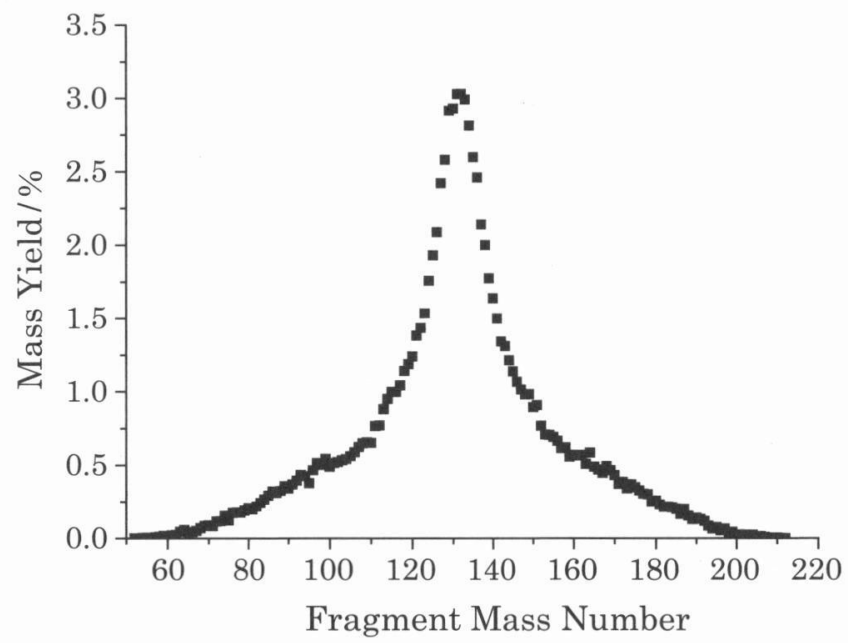

Figure 4. Distribution of the mass number of the fission fragments for the fission of ${ }^{264} \mathrm{Fm}$ at the excitation energy $E_{\mathrm{X}}=10.0 \mathrm{MeV}$.

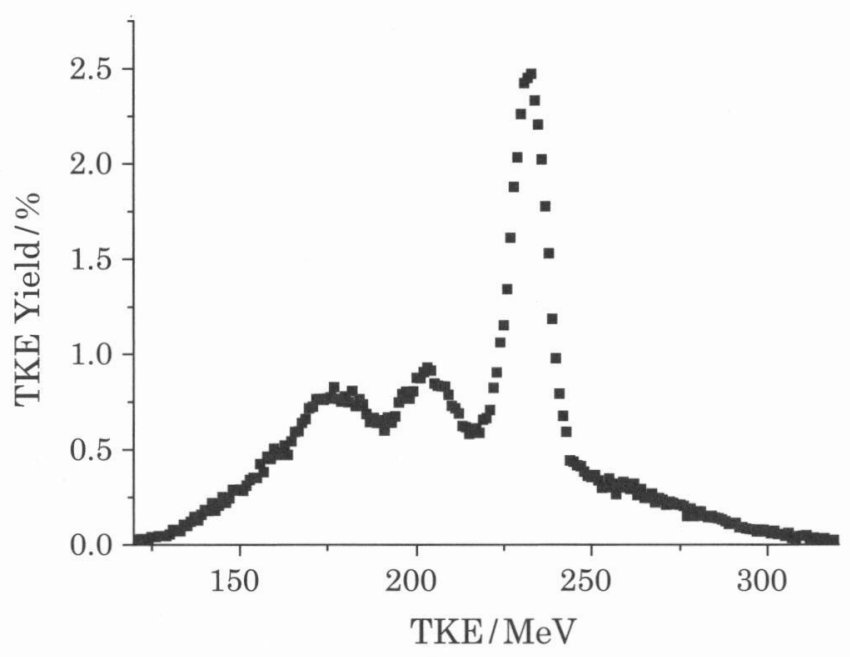

Figure 5. Distribution of the TKE of the fission fragments for the fission of ${ }^{264} \mathrm{Fm}$ at the excitation energy $E_{\mathrm{X}}=10.0 \mathrm{MeV}$. shown in Figure 5 is decomposed into these three components. It is seen that Component I corresponds to the events that belong to the highest TKE peak, Component III to the lowest TKE peak, and Component II lies in-between. As for the TKE distribution, we can thus understand that the structure seen in Figure 5 corresponds to the classification in the $\delta$-space.

The next problem is the mass distribution of each component classified by the value of $\delta$ at the scission. In Figure 8 ,

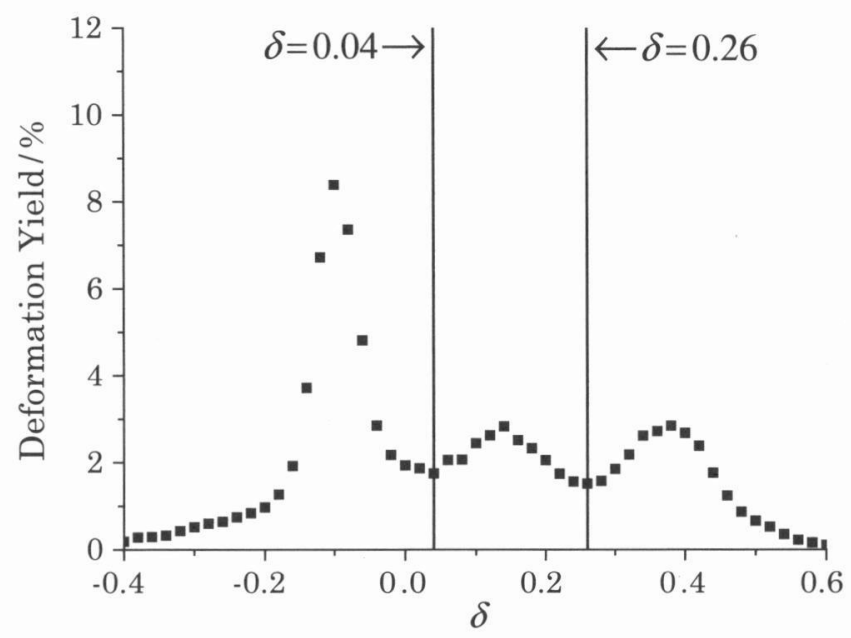

Figure 6. Distribution of the deformation parameter $\delta$ at the scission for the fission of ${ }^{264} \mathrm{Fm}$ at the excitation energy $E_{\mathrm{X}}=10.0 \mathrm{MeV}$.

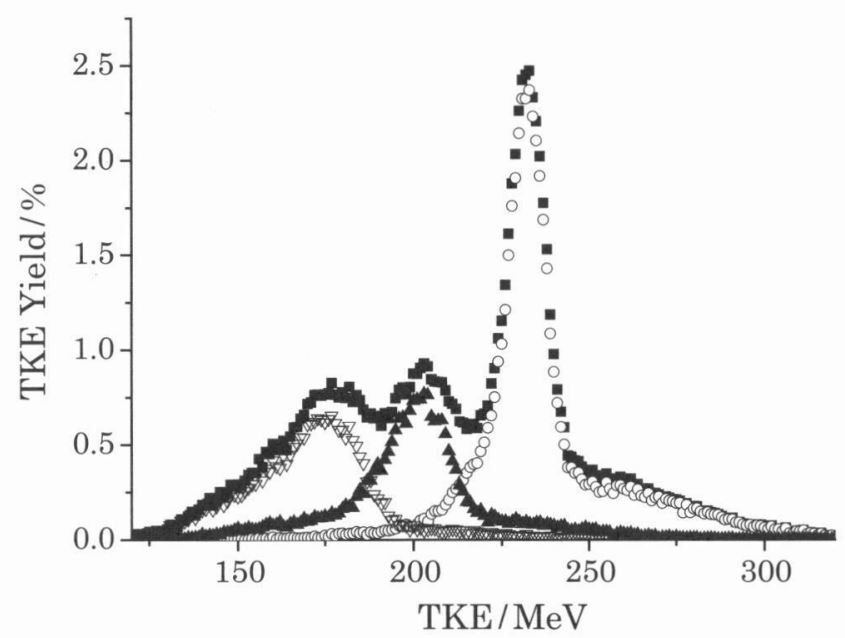

Figure 7. Decomposition of the TKE distribution according to the three components of the deformation parameter $\delta$. Open circles denote Component I, solid upward triangles Component II, and open downward triangles Component III. Solid squares denote the sum of the three components.

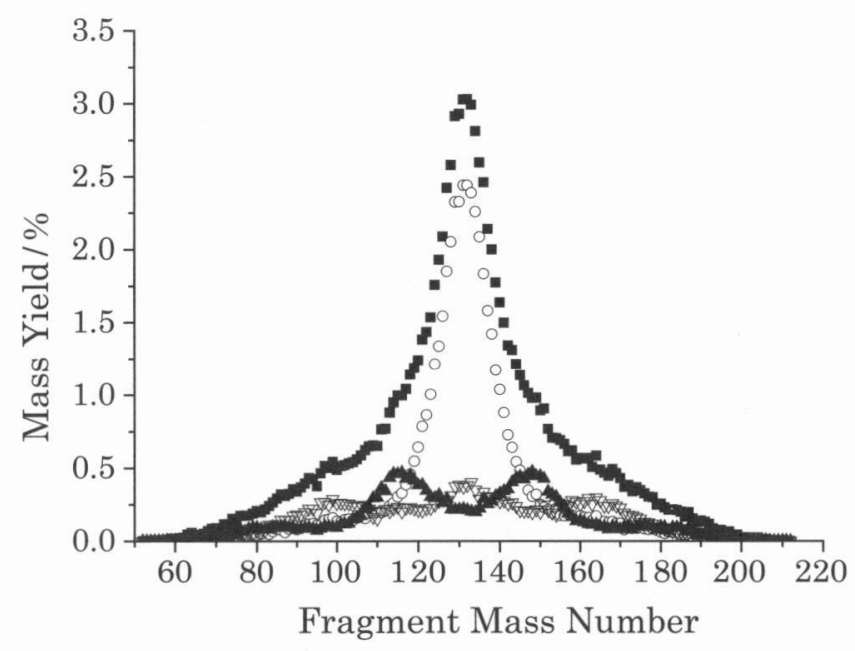

Figure 8. Decomposition of the mass distribution according to the three components of the deformation parameter $\delta$. The meaning of each symbol is the same as that in Figure 7. 
the total mass distribution is decomposed into the three components. It is found that Component I and Component III correspond to the mass-symmetric fission and Component II to the mass-asymmetric fission.

In order to study the origin of two components for the masssymmetric peak and one mass-asymmetric peak, we investigate the PES. First, Figure 9 shows the PES for the mass-symmetric fission of ${ }^{264} \mathrm{Fm}$. One can see that the ground state locates at $R_{\text {C.M. }} / 2 R \sim 0.45, \delta \sim 0.10(V \sim-0.70 \mathrm{MeV})$. It is found that there are two fission paths; one leads to Component I and the other to Component III. The path for Component I has only one saddle point $\left(R_{\text {C.M. }} / 2 R \sim 0.50, \delta \sim 0.25, V \sim 3.00 \mathrm{MeV}\right)$, while that for Component III has the second minimum $\left(R_{\text {C.M. }} / 2 R \sim 0.62, \delta \sim 0.30, V \sim-1.00 \mathrm{MeV}\right)$ and the second saddle point $\left(R_{\text {C.M. }} / 2 R \sim 0.70, \delta \sim 0.30, V \sim-0.10 \mathrm{MeV}\right)$. Therefore, the yield of Component $\mathrm{I}$ is expected to be greater than that of Component III.

Next, Figure 10 shows the PES in the case of $\delta=0.135$ which corresponds to the deformation peak of Component II. We find the mass-asymmetric second saddle point $\left(R_{\mathrm{C} . \mathrm{M}} / 2 R \sim\right.$ $0.70, A_{\mathrm{H}} \sim 147$ ), while we find only a mass-symmetric fission valley after the second saddle. It is difficult to predict where the peak of the mass distribution will locate only from this

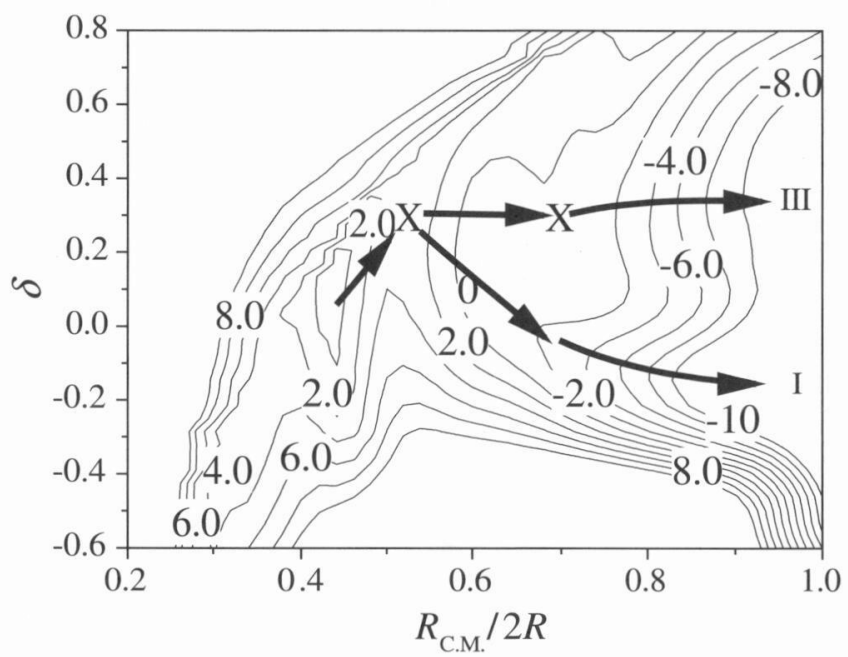

Figure 9. The contour plot of the PES for the mass-symmetric fission of ${ }^{264} \mathrm{Fm}$. The abscissa denotes the distance between the centers of mass of future fission fragments and the ordinate the deformation parameter $\delta . R$ is the radius of the parent nucleus. The first and the second saddle points are marked by X's. Arrows show expected fission paths

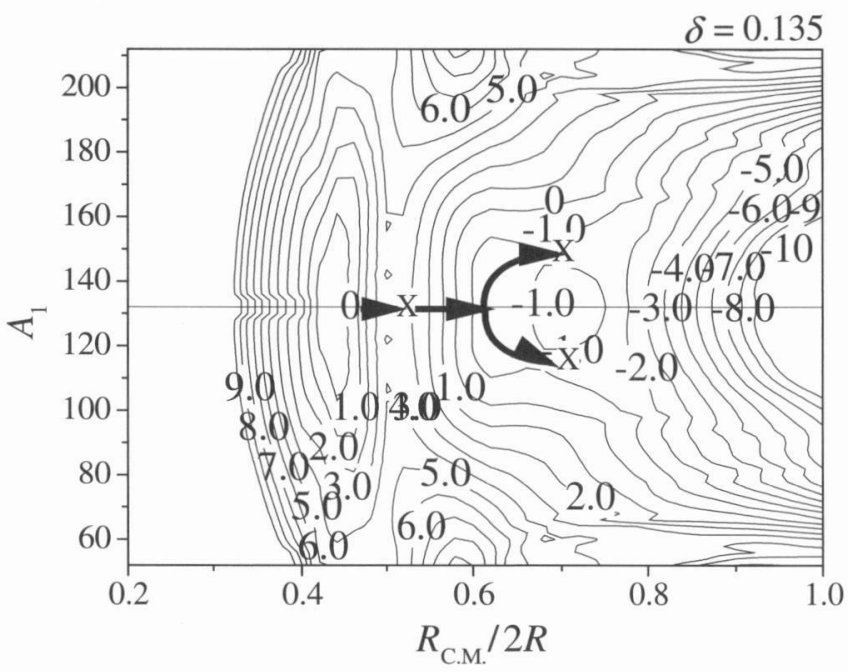

Figure 10. The contour plot of the PES in the case of $\delta=0.135$ for ${ }^{264} \mathrm{Fm}$. The abscissa denotes the distance between the centers of mass of future fission fragments and the ordinate the mass number of a fission fragment. The solid line shows the mass-symmetric division. $R$ is the radius of the fissioning nucleus. Arrows show expected fission paths.
PES. Thus we need the dynamical calculation. In comparison, Figure 11 shows the PES in the case of $\delta=-0.11$ which corresponds to the deformation peak of Component I. In this case, one finds the mass-symmetric saddle point and the masssymmetric fission valley.

Zhao et al. investigated the systematics of the TKE in the actinide region. ${ }^{10,11}$ They assumed that the TKE is equal to the Coulomb energy at the scission configuration for point charges, namely TKE $=Z_{1} Z_{2} e^{2} / D$, and defined the shape elongation at the scission point by $\beta=D /\left(R_{1}+R_{2}\right)$, where $R_{1,2}$ are the radii of fission fragments. They showed that there are mainly two types of scission configuration: a mass-asymmetric $\left(A_{\mathrm{H}}=140\right)$ configuration with $\beta=1.53$ and a mass-symmetric elongated configuration with $\beta=1.65$. Additionally, there is a mass-symmetric compact configuration with $\beta=1.33$ in the heavy region where the mass number of the fissioning nucleus is nearly equal to 260 . In order to compare our results with this experimental systematics, we calculate the peak values and the widths of the mass number and TKE distributions for each component from the Gaussian fitting. The results are listed in Table 1. One finds that the shape elongation $\beta$ of Component I corresponds to the mass-symmetric compact configuration, that of Component II to the mass-asymmetric $\left(A_{\mathrm{H}}=140\right)$ configuration and that of Component III to the masssymmetric elongated configuration. However, the mass number of the heavier fragment in the mass-asymmetry mode is predicted to be 147 and is different from the experimental value $A_{\mathrm{H}}=140$. Furthermore, our shape elongation $\beta$ is slightly bigger than that of the experimental systematics for the mass-symmetric elongated configuration.

The fermium nucleus ${ }^{264} \mathrm{Fm}$ has not been studied experimentally, but other fermium isotopes ${ }^{256,258} \mathrm{Fm}$ have been studied. A remarkable isotope dependence is observed experimentally; in the spontaneous fission, the mass-symmetric peak is observed for ${ }^{258} \mathrm{Fm}$, while the mass-asymmetric peak is

TABLE 1: Peak Values and Widths of the Mass and TKE Distributions Obtained from the Gaussian Fitting to Each Component

\begin{tabular}{lccccc}
\hline & $\left\langle A_{1}>\right.$ & $\sigma\left(A_{1}\right)$ & $\begin{array}{c}<\mathrm{TKE}> \\
/ \mathrm{MeV}\end{array}$ & $\begin{array}{c}\sigma(\mathrm{TKE}) \\
/ \mathrm{MeV}\end{array}$ & $\beta$ \\
\hline Component I & 131.5 & 6.80 & 232.1 & 6.30 & 1.31 \\
Component II & 147.0 & 9.10 & 200.8 & 10.4 & 1.50 \\
Component III & 132.3 & 34.2 & 171.7 & 15.0 & 1.77 \\
\hline
\end{tabular}

$\left\langle A_{1}>\right.$ : the peak mass number, $\sigma\left(A_{1}\right)$ : the variance of the mass number, $\langle$ TKE $>$ : the peak TKE, $\sigma(\mathrm{TKE})$ : the variance of the TKE, $\beta$ : the shape elongation deduced from $<A_{1}>$ and $<\mathrm{TKE}>$.

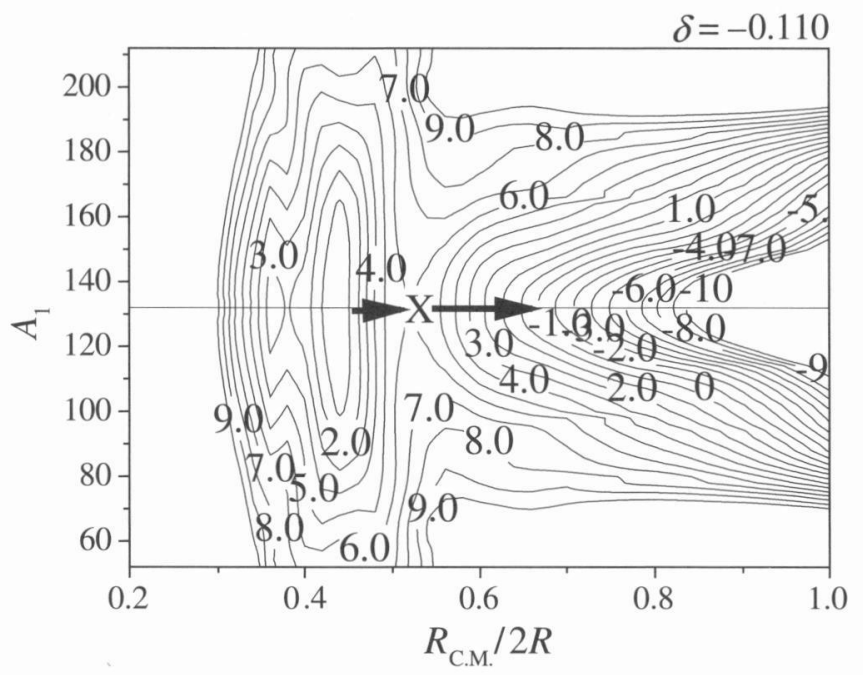

Figure 11. The same as Figure 10, but for the case of $\delta=-0.11$. 


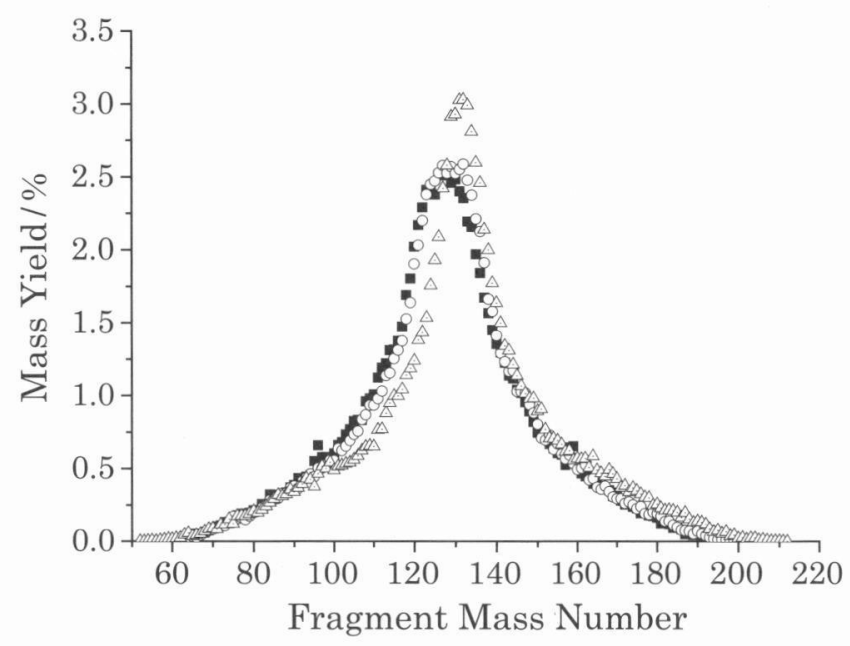

Figure 12. Isotope dependence of the mass distribution for the fission of ${ }^{256,258,264} \mathrm{Fm}$ at the excitation energy $E_{\mathrm{X}}=10.0 \mathrm{MeV}$. The solid squares denote the mass distribution of the fission fragments of ${ }^{256} \mathrm{Fm}$, open circles that of ${ }^{258} \mathrm{Fm}$, and open triangles that of ${ }^{264} \mathrm{Fm}$.

observed for ${ }^{256} \mathrm{Fm} .{ }^{1-6}$ We calculate the mass distributions for ${ }^{256,258} \mathrm{Fm}$ in the same manner as ${ }^{264} \mathrm{Fm}$. We compare the mass distributions of ${ }^{256,258,264} \mathrm{Fm}$ in Figure 12. When we compare the full width at half maximum (FWHM) for each mass distribution, this value increases from ${ }^{264} \mathrm{Fm}(\mathrm{FWHM}=25)$ to ${ }^{258} \mathrm{Fm}$ $(\mathrm{FWHM}=30)$ and then further to ${ }^{256} \mathrm{Fm}(\mathrm{FWHM}=33)$. We did not find the drastic isotope dependence that was found in the spontaneous fission. It should be noted that our calculation has been performed for the excitation energy $E_{\mathrm{X}}=10.0 \mathrm{MeV}$ and that mass-symmetric peaks have been observed in the thermal neutron induced fission of both ${ }^{255} \mathrm{Fm}$ and ${ }^{257} \mathrm{Fm}$.

\section{Summary}

Multi-modal nuclear fission of the fermium nuclei ${ }^{256,258,264} \mathrm{Fm}$ at the low excitation energy was dynamically investigated. We payed special attention to the deformation parameter $\delta$ which essentially controls the scission configuration. We classified the fission events in three components according to the values of the deformation parameter $\delta$ at the scission configuration: Component I $(\delta<0.04)$, Component II $(0.04<\delta<0.26)$ and Component III $(\delta>0.26)$. The mass and TKE distributions are well classified by the $\delta$ decomposition. It was found that the shape elongation $\beta$ which is deduced from the peak mass number and the peak TKE for each component is consistent with Zhao's experimental systematics. As a conclusion, we emphasize the importance of the deformation parameter $\delta$ at the scission configuration in the dynamical calculation. We are planning to extend this calculation to the four-dimensional one, where the deformation of the two fission fragments is expressed not by a single parameter $\delta$ but by two independent parameters $\delta_{1}$ and $\delta_{2}$.

Acknowledgements. The authors wish to express their gratitude to Dr. Y. Nagame and Dr. Y. L. Zhao for their useful discussions.

\section{References}

(1) R. C. Ragaini, E. K. Hulet, R. W. Lougheed, and J. Wild, Phys. Rev. C9, 399 (1974).
(2) W. John, E. K. Hulet, R. W. Lougheed, and J. J. Wesolowski, Phys. Rev. Lett. 27, 45 (1971).

(3) J. P. Balagna, G. P. Ford, D. C. Hoffman, and J. D. Knight, Phys. Rev. Lett. 26, 145 (1971).

(4) E. K. Hulet, J. F. Wild, R. J. Dougan, R. W. Lougheed, J. H. Landrum, A. D. Dougan, M. Schädel, R. L. Hahn, P. A. Baisden, C. M. Henderson, R. J. Dupzyk, K. Sümmerer, and G. R. Bethune, Phys. Rev. Lett. 56, 313 (1986).

(5) E. K. Hulet, J. W. Wild, R. J. Dougan, R. W. Lougheed, J. H. Landrum, A. D. Dougan, P. A. Baisden, C. M. Henderson, and R. J. Dupzyk, Phys. Rev. C40, 770 (1989).

(6) H. C. Britt, D. C. Hoffman, J. van der Plicht, J. B. Wilhelmy, E. Cheifetz, R. J. Dupzyk, and R. W. Lougheed, Phys. Rev. C30, 559 (1984).

(7) M. G. Itkis, V. N. Okolovich, and A. Ya. Rusanov, Z. Phys. A320, 433 (1985).

(8) Y. Nagame, I. Nishinaka, K. Tsukada, Y. Oura, S. Ichikawa, H. Ikezoe, Y. L. Zhao, K. Sueki, H. Nakahara, M. Tanikawa, T. Ohtsuki, H. Kudo, Y. Hamajima, K. Takamiya, and Y. H. Chung, Phys. Lett. B387, 26 (1996).

(9) Y. Nagame, Y. L. Zhao, I. Nishinaka, S. Goto, D. Kaji, M. Tanikawa, K. Tsukada, M. Asai, H. Haba, M. Sakama, S. Ichikawa, K. Sueki, H. Kudo, and H. Nakahara, Radiochim. Acta 78, 3 (1997).

(10) Y. L. Zhao, I. Nishinaka, Y. Nagame, M. Tanikawa, K. Tsukada, S. Ichikawa, K. Sueki, Y. Oura, H. Ikezoe, S. Mitsuoka, H. Kudo, and H. Nakahara, Phys. Rev. Lett. 82 , 3408 (1999).

(11) Y. L. Zhao, Y. Nagame, I. Nishinaka, K. Sueki, and H. Nakahara, Phys. Rev. C62, 014612 (2000).

(12) P. Möller, J. R. Nix, and W. J. Swiatecki, Nucl. Phys. A469, 1 (1987).

(13) V. V. Pashkevich, Nucl. Phys. A477, 1 (1988).

(14) T. Wada, Y. Abe, and N. Carjan, Phys. Rev. Lett. 70, 3538 (1993).

(15) T. Ichikawa, Doctoral thesis, Konan University (2003).

(16) Y. Aritomo and M. Ohta, Phys. Atom. Nucl. 66, 1105 (2003).

(17) Y. Abe, S. Ayik, P. -G. Reinhard, and E. Suraud, Phys. Rep. 275, 49 (1996).

(18) P. Fröbrich and I. I. Gontchar, Phys. Rep. 292, 131 (1998).

(19) K. T. R. Davis, A. J. Sierk, and J. R. Nix, Phys. Rev. C13, 2385 (1976).

(20) J. Randrup and W. J. Swiatecki, Nucl. Phys. A429, 105 (1984).

(21) H. J. Krappe, J. R. Nix, and A. J. Sierk, Phys. Rev. C20, 992 (1979).

(22) V. M. Strutinsky, Nucl. Phys. A95, 420 (1967).

(23) V. M. Strutinsky, Nucl. Phys. A122, 1 (1968).

(24) D. Scharnweber, W. Greiner, and U. Mosel, Nucl. Phys. A164, 257 (1971).

(25) J. Maruhn and W. Greiner, Z. Phys. 251, 431 (1972).

(26) S. Suekane, A. Iwamoto, S. Yamaji, and K. Harada, JAERI-memo, 5918 (1974).

(27) A. Iwamoto, S. Yamaji, S. Suekane, and K. Harada, Prog. Theor. Phys. 55, 115 (1976).

(28) M. Bolsterli, E. O. Fiset, J. R. Nix, and J. L. Norton, Phys. Rev. C5, 1050 (1972).

(29) A. V. Ignatyuk, G. N. Smirenkin, and A. S. Tishin, Sov. J. Nucl. Phys. 21, 255 (1975).

(30) K. Sato, S. Yamaji, K. Harada, and S. Yoshida, Z. Phys. A290, 149 (1979). 